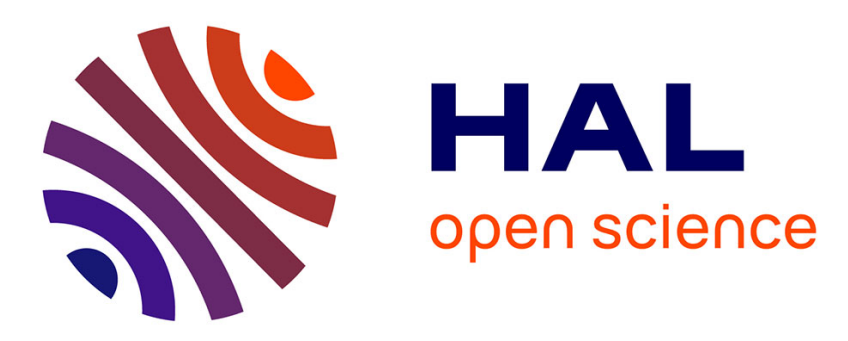

\title{
Separable scatter model of the detector and object contributions using continuously thickness-adapted kernels in CBCT
}

\author{
Navnina Bhatia, David Tisseur, Solène Valton, Jean Michel Létang
}

\section{To cite this version:}

Navnina Bhatia, David Tisseur, Solène Valton, Jean Michel Létang. Separable scatter model of the detector and object contributions using continuously thickness-adapted kernels in CBCT. Journal of X-Ray Science and Technology, 2016, 24 (5), pp.723-732. 10.3233/XST-160583 . hal-01438209

\author{
HAL Id: hal-01438209 \\ https://hal.science/hal-01438209
}

Submitted on 7 Jan 2019

HAL is a multi-disciplinary open access archive for the deposit and dissemination of scientific research documents, whether they are published or not. The documents may come from teaching and research institutions in France or abroad, or from public or private research centers.
L'archive ouverte pluridisciplinaire HAL, est destinée au dépôt et à la diffusion de documents scientifiques de niveau recherche, publiés ou non, émanant des établissements d'enseignement et de recherche français ou étrangers, des laboratoires publics ou privés. 


\title{
Separable scatter model of the detector and object contributions using continuously thickness-adapted kernels in CBCT
}

\author{
Navnina Bhatia ${ }^{\mathrm{a}, *}$, David Tisseur ${ }^{\mathrm{a}}$, Solene Valton ${ }^{\mathrm{b}}$ and Jean Michel Létang ${ }^{\mathrm{c}}$ \\ ${ }^{\text {a } C E A, ~ L I S T, ~ G i f-s u r-Y v e t t e, ~ F r a n c e, ~}$ \\ ${ }^{\mathrm{b}} R X$ Solutions, 27 rue Saturne, Chavanod, France \\ ${ }^{\mathrm{c}}$ Univ Lyon, INSA Lyon, Université Lyon 1, UJM-Saint Etienne, CNRS, Inserm, Centre Léon Bérard, \\ CREATIS UMR 5220 U1206, Lyon, France
}

\begin{abstract}
Due to the increased cone beam coverage and the introduction of flat panel detector, the size of X-ray illumination fields has grown dramatically in Cone Beam Computed Tomography (CBCT), causing an increase in scatter radiation. Existing reconstruction algorithms do not model the scatter radiation, so scatter artifacts appear in the reconstruction images. The contribution of scattering of photons inside the detector itself becomes prominent and challenging in case of X-ray source of high energy (over a few $100 \mathrm{keV}$ ) which is used in typical industrial Non Destructive Testing (NDT). In this paper, comprehensive evaluation of contribution of detector scatter is performed using continuously thickness-adapted kernels. A separation of scatter due to object and the detector is presented using a four-Gaussian model. The results obtained prove that the scatter correction only due to the object is not sufficient to obtain reconstruction image free from artifacts as the detector also scatters considerably. The obtained results are also validated experimentally using a collimator to remove the contribution of object scatter.
\end{abstract}

Keywords: CBCT, scatter artifact correction, CIVA, industrial NDT

\section{Introduction}

One of the well-recognized challenge of cone-beam computed tomography (CBCT) is the presence of scatter contamination within the projection images. Existing reconstruction algorithms do not model the scatter radiation. Therefore scatter artifact appears on the reconstruction images as shading, cupping effect, reduced contrast etc. This leads to overall inaccuracies in the reconstruction values.

Majority of the scatter correction techniques focus on scatter contribution from the object only. But recent studies [1] [2] [3] have proved that in the high energy range used in industrial Non-Destructive Testing (NDT), the contribution of detector scatter also becomes important. While evaluating the role of detector in the reconstruction artifacts, a number of scattering and blurring processes within the detector need to be taken into account.

Firstly, X-ray radiation can be Compton scattered by any part of the detector surroundings like mountings, back plate or even the detector layer itself [1]. Bub et al [1] show that this scattering

\footnotetext{
${ }^{*}$ Corresponding author: Navnina Bhatia, CEA, LIST, F-91191, Gif-sur-Yvette, France. Tel.: +33 169 087863 ; E-mail: navnina.bhatia@cea.fr.
} 
can also lead to the edge blurring of the object in the obtained projections. The part of the detector directly exposed to full beam, produces more Compton scattering than the part of the detector under the shadow of the highly attenuating object. This is because of attenuation of the primary intensity under the object. As a result the sharpness of the edges of the object appear degraded. This can cause additional grey levels appearing in the reconstruction leading to deformations. Another important effect at high $\mathrm{X}$ ray energy source is the backscattering of photons from the material behind the detector [4]. If the scintillator layer is made of low absorbing material, a large part of radiation can be transmitted to the back-plate. For the energies above $\mathrm{K}$ edge of the back plate, there is appreciable increase in backscattering by the production of low energy fluorescence X rays. These backscattered photons may be reabsorbed at a different site than the first interaction site. As a result the overall spatial resolution of the detector decreases.

Additionally, when X-rays interact with a phosphor (by most pronounced photo-electric effect), $\mathrm{X}$-ray fluorescence photons are produced which may be reabsorbed by the detector areas adjacent to the primary $\mathrm{x}$-ray interaction site. This may cause a loss of spatial resolution and an increase in image noise [5] [6]. Moreover, the fluorescence optical photon may also scatter within the scintillator layer which causes blurring, also known as veiling glare [7] [8]. Its magnitude mainly depends on the thickness and material characteristics of the detection layer.

In this article, we propose to evaluate the contribution of detector scattering at NDT energy range using continuously thickness adapted Scatter Kernel Superposition (SKS) method [9]. The continuous method takes into account better sampling of the kernels with respect to the thickness of the object to get an accurate model of variability in shape and in amplitude of the scatter kernels over the whole thickness range. However, the continuous method and other classical SKS method [10] use a twoGaussian model for the fitting of kernels. This two-Gaussian parametrization of kernels has several shortcomings. Firstly, the scatter contributions from the detector and the object are entangled, which makes the parameter fitting of the continuous model tricky. Moreover, the high frequency contribution of the detector scatter generates a strong peak in the center pixel of the detector where the pencil beam is impinged. This shape calls for the necessity to explore an increase in order of the Gaussian model. Therefore, we propose in this paper, by means of both experiments and simulations, to identify the respective scatter contributions of the object and detector using a four-Gaussian model to have a more stable modeling.

We begin by outlining the four-Gaussian analytic description of the kernels. Subsequently, the CBCT acquisition set up is described in detail. An in-depth analysis of the four-Gaussian contributions is carried out in Section 3. Experimental validation is finally performed with both fan beam and cone beam geometries.

\section{Method and materials}

\subsection{Four-Gaussian model}

In the SKS scatter correction approach with continuously thickness-adapted kernels [9], the scatter signal can be modeled as the sum of the scatter contributions from a group of pencil beams passing through the object and the detector. For each pencil beam input, a resulting kernel which has the weight of the scatter to primary ratio is determined. The total scatter signal $S(m, n)$ with $m$ and $n$ as the pixel position on the detector, can then be modeled as:

$$
S(m, n)=\sum_{k} \sum_{l} P(k, l) h_{T(k, l)}(m-k, n-l)
$$


where, $h_{T}$ is the thickness $(T)$ dependent kernel, with amplitude equal to the ratio of the scattered signal at the current pixel to the primary signal, at the pencil beam centered pixel.The sum in the convolution process runs over all pixels $(k, l)$ of the detector. $P$ is the primary signal contributed by the photons passing directly without any attenuation. Instead of using Beer-Lambert law based on an effective linear attenuation coefficient [10], the thickness $T(k, l)$ in Equation 2 is directly computed from a look-up table $f_{B H}$ - computed during the calibration stage of the kernels - that relates the true slab thickness with respect to the transmittance

$$
T(k, l)=f_{B H}\left(\frac{P(k, l)}{O(k, l)}\right)
$$

where $O$ is the unattenuated image. It is worthy to note that the beam-hardening is taken care of with this procedure. The acquisition parameters and the material of the calibration slabs must be the same as the object under study. Value of directly transmitted primary fluency $P$ were calculated for each thickness of the simulated slab. The thickness in terms of the transmittance $P(k, l) / O(k, l)$ is calculated as described in Section 3.1.

The pencil beam kernel $h_{T}$ can be fitted into the equation formed by four circularly symmetric Gaussian functions describing the shape of the kernel:

$$
\begin{aligned}
h_{T}(m-k, n-l)= & A \exp \left(-\frac{(m-k)^{2}+(n-l)^{2}}{2 \sigma_{1}^{2}}\right)+B \exp \left(-\frac{(m-k)^{2}+(n-l)^{2}}{2 \sigma_{2}^{2}}\right) \\
& +C \exp \left(-\frac{(m-k)^{2}+(n-l)^{2}}{2 \sigma_{3}^{2}}\right)+D \exp \left(-\frac{(m-k)^{2}+(n-l)^{2}}{2 \sigma_{4}^{2}}\right)
\end{aligned}
$$

The four-Gaussian model is necessary in order to clearly separate the contributions of the object and of the detector.

\subsection{Kernel generation and fitting}

Monte Carlo (MC) simulations were performed in the CT module of CIVA software [11] for the generation of kernels. Pencil beam source was impinged on slabs of same material as the object under study and discrete set of point spread 2D kernels were obtained on the flat panel detector.

The flat panel was modeled in a standard multi-layer representation consisting of $0.1 \mathrm{~mm}$ aluminium front layer followed by air-gap of $0.8 \mathrm{~mm}$, a $0.06 \mathrm{~mm}$ CsI layer, $1 \mathrm{~mm}$ silicon substrate and finally a $1 \mathrm{~mm}$ lead back plate.

The eight parameters of Equation 3, $A, B, C, D, \sigma_{1}, \sigma_{2}, \sigma_{3}, \sigma_{4}$ are also interpolated in terms of thickness to obtain a continuous kernel map with respect to thickness [9]. We have used a fourGaussian model in order to separate the contribution of object and detector. To get realistic kernels and in particular to take into account optical processes in the detector, real acquisitions on slabs of various thickness could also be advantageously used in practice with an X-ray pencil beam geometry.

\subsection{Acquisition set up with aluminum turbo}

For the acquisition set up, the source to detector distance was $845 \mathrm{~cm}$ and the distance between source and axis of rotation was at $424 \mathrm{~cm}$. The set up mainly consisted of a X-ray source, an object rotational table and a flat panel detector. The X-ray source unit of maximum $230 \mathrm{kV}$ was used. A 


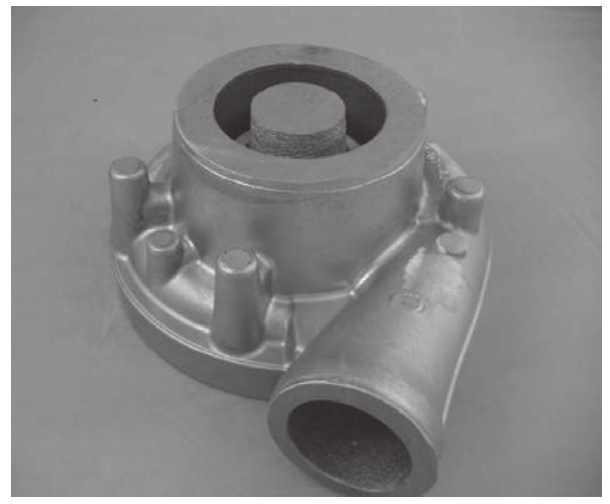

Fig. 1. Picture of sample of the aluminum turbo.

copper filter of $0.35 \mathrm{~mm}$ was used in the spectrum. Flat panel detector used had a size $1840 \times 1456$ with $2 \times 2$ binning size and $0.254 \mathrm{~mm}$ pixel size.

Acquisitions were performed on an aluminum turbo as given in Fig. 1 which had a maximum dimension of around $300 \mathrm{~mm}$.

\subsection{Acquisition set-up with collimator slit}

In order to validate our results with experimental results, acquisitions were performed with a collimator slit inserted in between the object and the detector. The set up is described in Fig. 2. The collimator slit was made of steel with a thickness of $30 \mathrm{~mm}$ and width $15 \mathrm{~mm}$. The introduction of the collimator removed approximately all the contribution of the object scatter.

\section{Results}

\subsection{Analytical fitting of calculation of thickness}

As discussed in Section 2.1, in order to calculate the thickness at each pixel, simulation were performed with slabs of different thickness. The acquisition parameters and the material of the slabs was kept the same as the object under study. Value of directly transmitted primary fluency $P$ were calculated for each thickness of the simulated slab.

The thickness vs transmittance LUT $f_{B H}$ given in Equation 3 has been analytically calculated using least square fitting as shown in Fig. 4 to determine the coefficients $a_{1}, b_{1}, a_{2}, b_{2}$ in Equation 4 . The classical thickness determination based on the effective linear attenuation coefficient is also shown by the red curve in Fig. 4. The beam-hardening, although not really strong, is clearly visible. Equation 4 gives the relationship of thickness $T$ value in terms of transmittance $P(k, l) / O(k, l)$.

$$
\begin{aligned}
T(k, l) & =f_{B H}\left(\frac{P(k, l)}{O(k, l)}\right) \\
& =-a_{1} \ln \left(\frac{P(k, l)}{O(k, l)}\right)^{b_{1}}-a_{2} \ln \left(\frac{P(k, l)}{O(k, l)}\right)^{b_{2}}
\end{aligned}
$$

using $a_{1}=20.8, b_{1}=0.83, a_{2}=0.000572, b_{2}=1$ 


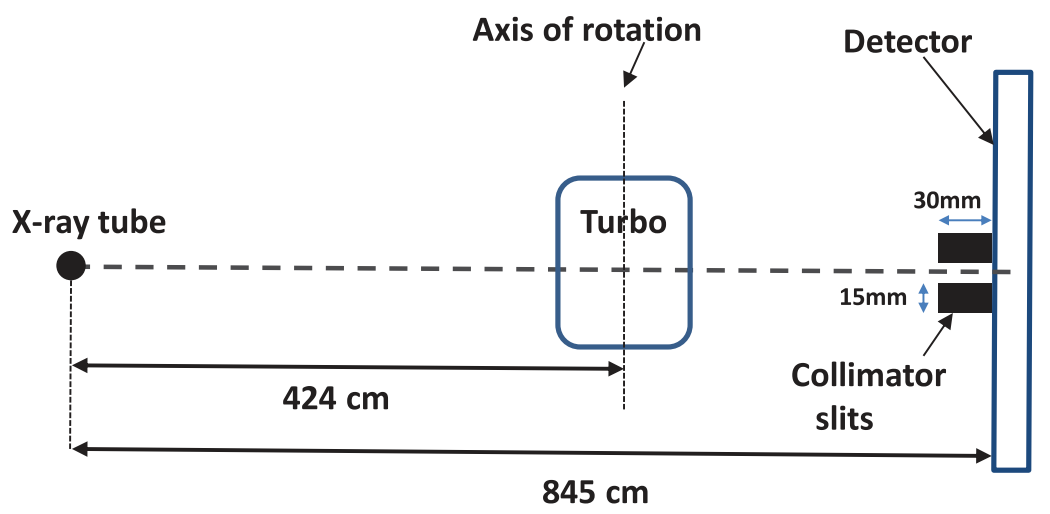

Fig. 2. Schematic of the acquisition set up with the collimator slit.

\subsection{Analytical fitting of kernels for continuous kernel map}

The fitting of parameters $A, B, C, D, \sigma_{1}, \sigma_{2}, \sigma_{3}, \sigma_{4}$ of the four-Gaussian model with respect to thickness using least square fitting is shown in Fig. 3. From the fitting of the parameters, we observe that the standard deviation $\sigma_{1}$ and $\sigma_{2}$ vary over the thickness of the object whereas $\sigma_{3}$ and $\sigma_{4}$ remain constant. We can draw inference that $\sigma_{1}$ and $\sigma_{2}$ give the the scattering contribution of the object which increases over the thickness of the object. Moreover, we notice that $\sigma_{1}$ is very far from $\sigma_{2}$. Henceforth, we analyze that $\sigma_{1}$ displays the low frequency contribution of multiple scattering inside the object, whereas $\sigma_{2}$ gives comparatively low order scattering contribution. We also mark not much difference in the parameters $A \& B$ which weight the amplitude of the shape of the Gaussians. This variation of parameters $A, B, \sigma_{1}, \sigma_{2}$, demonstrate that a two-Gaussian model for the object is necessary.

Similarly, observing $\sigma_{3}$ and $\sigma_{4}$ remain constant over the thickness of the object, we infer that $\sigma_{3}$ and $\sigma_{4}$ define the high frequency contribution from the detector. Following the same line of reasoning, $\sigma_{3}$ and $\sigma_{4}$ are different from each other whereas and parameters $C, D$ are not outlying each other. Therefore, a two-Gaussian model for the detector is also crucial.

\subsection{Scatter correction on aluminium turbo}

Figure 5 a) displays the reconstruction slice obtained with uncorrected projections using FDK algorithm. We first evaluated the scatter contribution of the object alone and we corrected the projections with only scatter kernels of the object. The result obtained is shown in Fig. 5 b). It clearly shows that the scatter correction due to only object is not sufficient as scatter artifacts are still visible in the reconstruction image. This is also demonstrated in Fig. 6 which displays the plot profile of the corrected and uncorrected reconstruction slices. The scatter kernels of detector and object were then used to perform the corrections of the projections. The reconstruction obtained with this correction is shown in Fig. $5 \mathrm{c})$. The obtained result with the scatter correction of object and detector is in agreement with the expected value of effective linear attenuation coefficient at mean energy $98 \mathrm{keV}(0.48$ per $\mathrm{cm})$.

Table 1 displays the mean and standard deviation of the reconstructed values for corrected and uncorrected data in air and aluminum region calculated using binary masks. Uncorrected value of $0.258 \mathrm{~cm}^{-1}$ for the linear attenuation coefficient of aluminum is estimated. By using only object scatter kernels we obtain a value of $0.324 \mathrm{~cm}^{-1}$ for the linear attenuation coefficient of aluminum. In the considered energy range, the value of linear attenuation constant per $\mathrm{cm}$ for mean energy 100 $\mathrm{keV}$ is $0.48 \mathrm{~cm}^{-1}$. The relative absolute error in percentage is $32.5 \%$ for the scatter correction with object kernels. Whereas, for detector and object kernels both we obtain a value of $0.510 \mathrm{~cm}^{-1}$ for the 

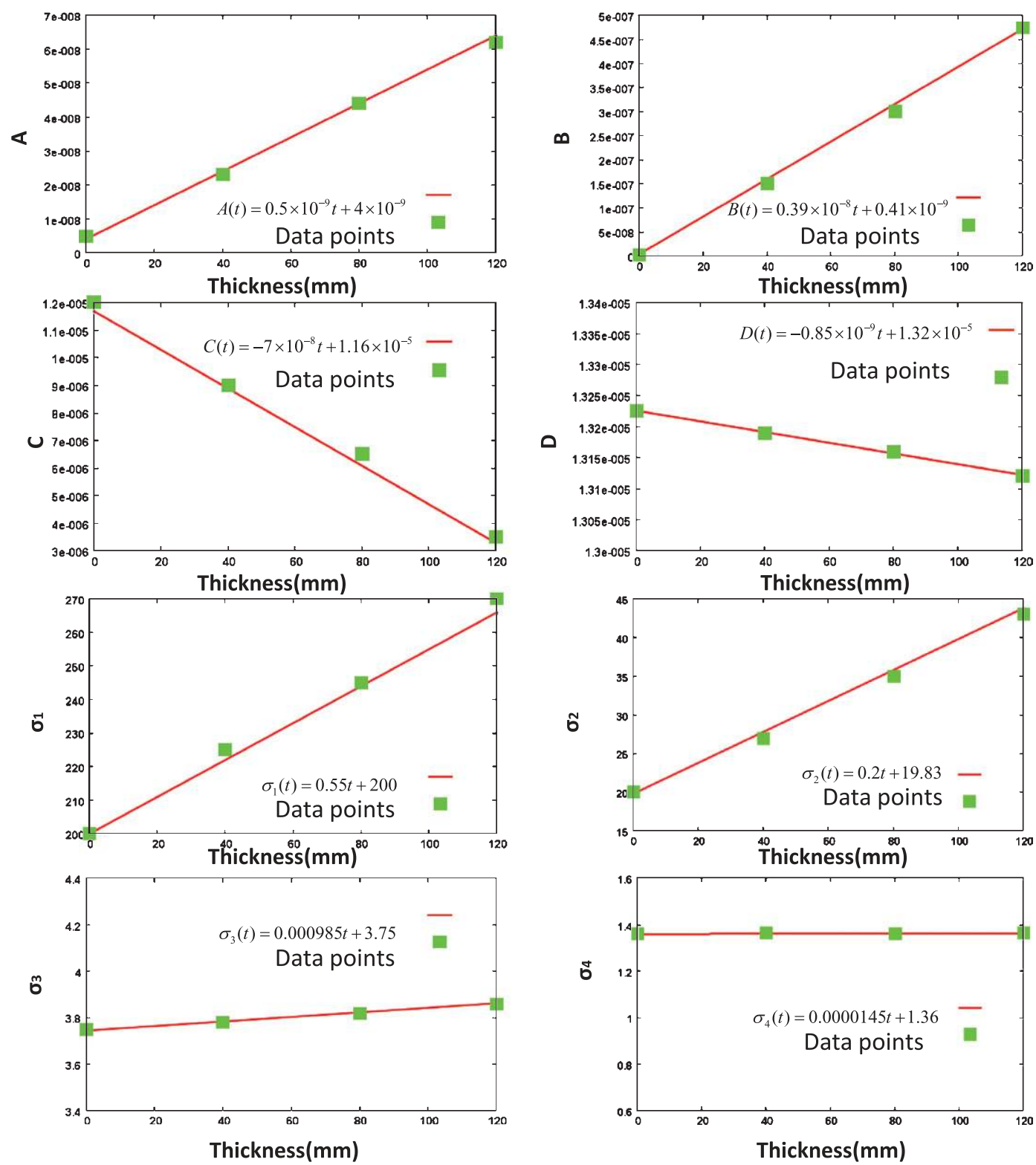

Fig. 3. Fitting of parameters $A, B, C, D, \sigma_{1}, \sigma_{2}, \sigma_{3}, \sigma_{4}$ with thickness.

aluminum. The relative absolute error is reduced to $6.25 \%$. The remaining artifact that we see in the scatter corrected reconstruction arise from the very high attenuation along the largest dimension of the object. The dynamic range of the detector was not high enough to take into account the attenuation along the highest dimension.

\subsection{Validation by experimental result using a collimator}

In order to validate the results obtained with the continuously thickness adapted SKS scatter correction algorithm, we performed acquisitions using a collimator made of steel with $30 \mathrm{~mm}$ thickness. 


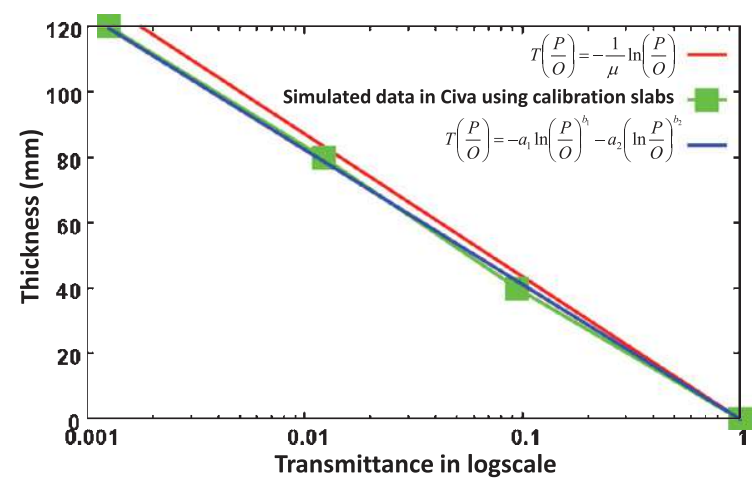

Fig. 4. Fitting of thickness Vs transmittance curve to obtain correct value of thickness in terms of transmittance.

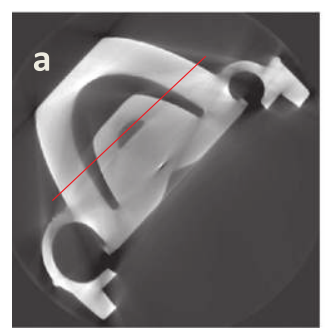

Uncorrected

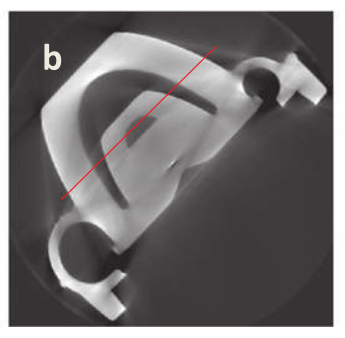

Only object Scatter correction

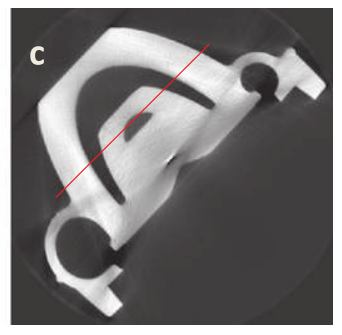

Detector and object Scatter correction

Fig. 5. Reconstruction slice with a) uncorrected projections b) corrected projections by only scatter kernels c) corrected projections by scatter and detector kernels.

The set up is described in the Section 2.4. Fan beam was produced and the contribution of the scatter from the object was eliminated by introducing a collimator slit between the object and the detector. All the other acquisition parameters were kept same as the previous set up without the collimator slit. The reconstruction was performed on the projections obtained with this set up and the result is shown in Fig. 7 a). It clearly shows the presence of scatter artifacts due to detector. We compare this experimental result with the result obtained by scatter correction performed by the algorithm using only object scatter kernels shown in Fig. 7 b). Figure 8 compares the plot profile of the experimental result with the collimator and scatter correction by the algorithm using object scatter kernels. The plot profiles demonstrate identical results validating the results obtained by the algorithm and proving considerable contribution of detector scatter.

Table 2 displays the mean and standard deviation of the reconstructed values for air $\&$ aluminum region for experimental result and correction with object scatter kernels. The linear attenuation coefficient obtained for aluminum and air is $0.312 \mathrm{~cm}^{-1}$ and $0.096 \mathrm{~cm}^{-1}$ respectively for the experimental result. This is in agreement with the scatter correction with object kernels result which is $0.324 \mathrm{~cm}^{-1}$ for aluminum and $0.106 \mathrm{~cm}^{-1}$ for air. In the considered energy range, the value of linear attenuation coefficient for mean value of spectrum $(100 \mathrm{keV})$ is $0.48 \mathrm{~cm}^{-1}$ and $0.0001 \mathrm{~cm}^{-1}$ for aluminum and air respectively. This result demonstrates that in the considered energy range, object scatter correction is not sufficient and the detector scatter contribution also needs to be taken into account to obtain correct reconstruction values. 
Table 1

Mean and standard deviation values for aluminum and air region for uncorrected and corrected reconstruction slices

\begin{tabular}{llcc}
\hline & & mean $\left(\mathrm{cm}^{-1}\right)$ & std. dev. \\
\hline Uncorrected & aluminium & 0.258 & \pm 0.0079 \\
& air & 0.098 & \pm 0.0054 \\
Object scatter correction & aluminium & 0.324 & \pm 0.0061 \\
& air & 0.106 & \pm 0.0056 \\
Object and detector scatter correction & aluminium & 0.510 & \pm 0.0078 \\
Mean spectrum value & air & 0.0008 & \pm 0.0036 \\
& aluminium & & 0.48 \\
\hline
\end{tabular}

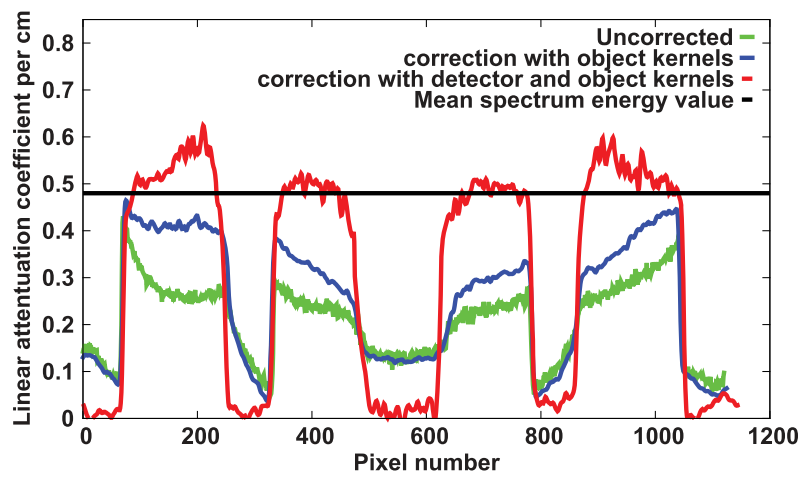

Fig. 6. Plot profile of uncorrected and corrected reconstruction slice by only scatter kernels and scatter and detector kernels.

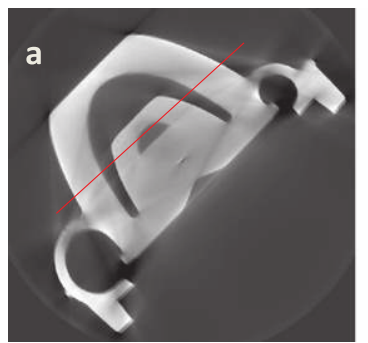

Experimental result with collimator

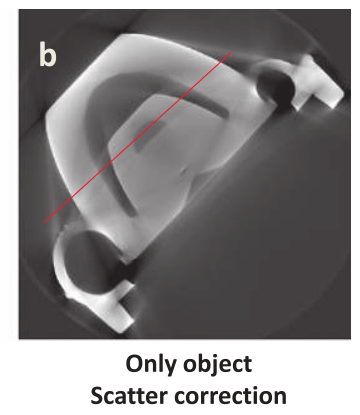

Scatter correction

Fig. 7. Reconstruction slice by a) experimental results with collimator b) corrected projections by only object scatter kernels.

\section{Discussion and conclusion}

The analytical description of the scatter kernels using four-Gaussian model separates the low frequency contribution of scatter due to object and the high frequency contribution of the detector. This separation of the contributions is necessary to clearly follow the effect of detector scatter on the reconstruction in CBCT by developing a separate model for the detector.

The object scatter itself requires a two-Gaussian model to take into account higher order and lower order scattering from the object. The amplitude parameters of scatter kernels $A \& B$ increase with 


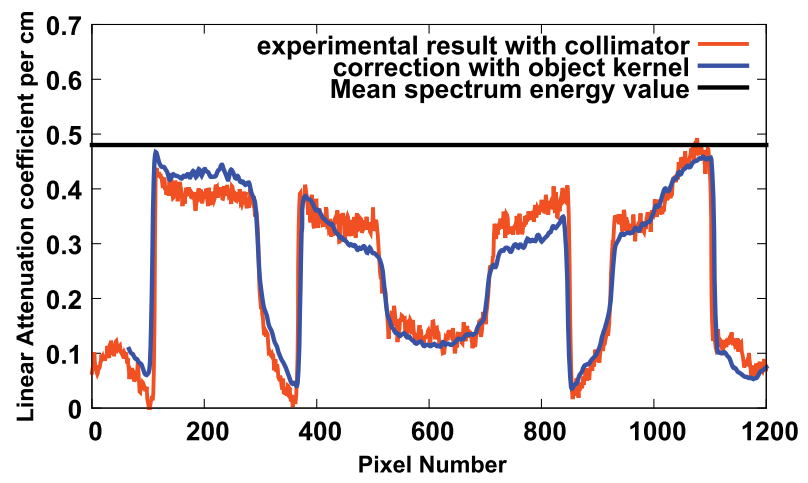

Fig. 8. Plot profile of experimental result with collimator and scatter correction with object scatter kernels.

Table 2

Mean and standard deviation values for aluminium and air region for experimental result with collimator and correction with object scatter kernels

\begin{tabular}{llcc}
\hline & & mean $\left(\mathrm{cm}^{-1}\right)$ & std.dev \\
\hline Experimental result with collimator & aluminium & 0.312 & \pm 0.0056 \\
& air & 0.096 & \pm 0.0023 \\
Correction with object scatter kernels & aluminium & 0.324 & \pm 0.0090 \\
& air & 0.106 & \pm 0.0067 \\
Theoretical values & aluminium & & 0.48 \\
& air & & 0.0001 \\
\hline
\end{tabular}

increase in thickness of the object because of the increase in SPR. $\sigma_{1}$ representing the very low frequency contributions from the object after multiple scattering of photons and $\sigma_{2}$ representing comparatively high frequency contribution from the object after fewer scattering of the photons, both increase with the thickness of the object. Similarly, the high frequency contribution of the detector model also requires a two-Gaussian model. The amplitude parameters for the detector Gaussian, $C \& D$, decrease with thickness. Due to higher attenuation, lesser primary intensity reaches the detector leading to fewer Compton scattering events in the detector and its surrounding. Also, D, which weights the high frequency detector contribution, remains constant while $\mathrm{C}$, which weights comparatively lower frequency detector contribution, decreases with respect to thickness. One explanation to this behavior could be that $\mathrm{C}$ is related to the back scattering from the back plate and since effective energy increases with respect to thickness, the scattering from the back plate becomes less and less back scattered. Whereas, D could be related to florescence in the scintillator, which remains constant over the thickness. The high frequency contribution of the detector given by $\sigma_{3}, \sigma_{4}$ remain constant over the thickness of the object.

Using this analytical description of the scatter kernels, the projections were corrected to perform a comprehensive study of the contributions of object and detector scattering. The correction of projections using only object scatter kernels produced reconstruction image with scatter artifact. The relative absolute error with respect to the expected value is $32.5 \%$ for the scatter correction with object kernels. The correction of projections using object and detector scatter kernels reduced the relative error to $6.25 \%$. The results obtained were validated with experimental results using a collimator. The relative absolute error between the experimental result using collimator and the scatter correction using object kernel was $3.7 \%$. 
The obtained result proves in this particular case study that the correction of the scatter due to the object only is not sufficient to completely remove scatter artifact in the reconstruction. The contribution of the detector scattering is very significant at the energy range used. We remark that the detector scatter depends on several parameters including the spectrum used and components (front filter, back plate etc.) configuration of the detector. The importance of the contribution of the detector scatter over the object scatter will therefore depend on each case study. Although the information about the component structure of the detector was only approximate in the simulation model of this study as it is generally kept anonymous by the manufacturer - the improvement in the scatter reduction using both object and detector scatter kernels is noteworthy. To further improve the realism of the detector kernels, either a more accurate 3D description of the detector could be used in the Monte Carlo simulation instead of a simple multi-layer model or direct measurements of the detector kernel could be carried out. Moreover, it could be additionally possible to implement a detector-specific tuning of the apodization function of the FDK reconstruction, for example, by measuring the noise power spectrum of the detector to take into account the detector variability of noise levels.

\section{References}

[1] A. Bub, S. Gondrom, M. Maisl, N. Uhlmann and W. Arnold, Image blur in a flat-panel detector due to compton scattering at its internal mountings, Measurement Science and Technology, 18(6) (2007), 1270-1277.

[2] P. Wils, J.M. Letang and J.P. Bruandet, Secondary radiations in cone-beam computed tomography: Simulation study, $J$ Electron Imaging 21(2) (2012), 021113.

[3] G. Poludniowski, P.M. Evans, A. Kavanagh and S. Webb, Removal and effects of scatterglare in cone-beam CT with an amorphous-silicon flat-panel detector, Phys Med Biol 56 (2011), 1837-1851.

[4] M. Hoheisel, A. Korn and J. Giersch, Influence of backscattering on the spatial resolution of semiconductor x-ray detectors, NIMB 546(12) (2005), 252-257.

[5] D. Nikolopoulos, I. Kandarakis, D. Cavouras, I. Valais, D. Linardatos, C. Michail, S. David, A. Gaitanis, C. Nomicos and A. Louizi, Investigation of radiation absorption and $\mathrm{x}$-ray fluorescence properties of medical imaging scintillators by monte carlo methods, NIMB 565(2) (2006), 821-832.

[6] J.M. Boone, J.A. Seibert, J.M. Sabol and M. Tecotzky, A monte carlo study of X-ray fluorescence in X-ray detectors, Med Phys 26(6) (1999), 905-916.

[7] R. Luhta and J.A. Rowlands, Origins of flare in x-ray image intensifiers, Med Phys 17(5) (1990), 913-921.

[8] L. Dimitrios and W.F. Jeffrey, Impact of flat panel-imager veiling glare on scatter-estimation accuracy and image quality of a commercial on-board cone-beam ct imaging system, Med Phys 39(9) (2012), 5639-5651.

[9] N. Bhatia, D. Tisseur, F. Buyens and J.M. Letang, Scattering correction using continuously thickness-adapted kernels, NDT \& E 78 (2016), 52-60.

[10] M. Sun and J.M. Star-Lack, Improved scatter correction using adaptive scatter kernel superposition. Phys Med Biol 55(22) (2010), 6675-6720.

[11] http://www-civa.cea.fr/ 